\title{
Strength of tensor forces from neutron drops in ab initio relativistic Brueckner-Hartree-Fock theory
}

\author{
Sibo Wang, ${ }^{1}$ Hui Tong, ${ }^{1}$ Pengwei Zhao $\odot,{ }^{1, *}$ and Jie Meng ${ }^{1,2,3}$ \\ ${ }^{1}$ State Key Laboratory of Nuclear Physics and Technology, School of Physics, Peking University, Beijing 100871, China \\ ${ }^{2}$ Department of Physics, University of Stellenbosch, Stellenbosch, South Africa \\ ${ }^{3}$ Yukawa Institute for Theoretical Physics, Kyoto University, Kyoto 606-8502, Japan
}

(Received 8 August 2019; revised manuscript received 18 October 2019; published 23 December 2019)

\begin{abstract}
The evolution of spin-orbit splittings of neutron drops along with the neutron number and its connection with the tensor-force strength have been investigated systematically for different external fields in the relativistic Brueckner-Hartree-Fock (RBHF) and relativistic Hartree-Fock (RHF) theories. Based on the RHF functional PKO1, it is found that a good consistency between the RBHF and RHF results for the total energies can be obtained only for those neutron drops whose central densities are close to the saturation density of nuclear matter. Nevertheless, by rescaling the density dependence of the RHF functional, the RBHF total energies of neutron drops in different external fields can be well reproduced. The optimized tensor-force strength $\lambda$ in the RHF theory, which reproduces the microscopic RBHF spin-orbit splittings, is running with the strength of the external fields of neutron drops. This provides an important guide for future determination of tensor forces in nuclear energy density functionals based on microscopic ab initio calculations.
\end{abstract}

DOI: 10.1103/PhysRevC.100.064319

\section{INTRODUCTION}

The covariant density functional theory (CDFT), with a few parameters, has achieved great success in describing the basic properties for most nuclei in the nuclear chart [1-7]. One of the frontiers in nuclear physics is to derive nuclear density functionals from $a b$ initio calculations in terms of the nucleon-nucleon $(N N)$ interactions [8,9]. Progress has been made in Ref. [10] by fitting to the isovector effective mass difference derived from the relativistic Brueckner-HartreeFock (RBHF) theory for nuclear matter. From relativistic bare $N N$ interactions, fully self-consistent RBHF theory for finite nuclei has been achieved [11,12], which provides an important guide for future microscopic derivations of nuclear density functionals. In particular, by applying RBHF theory to neutron drops, i.e., finite number of neutrons confined in an external field, a tensor-force effect on the evolution of spin-orbit (SO) splittings is revealed clearly $[13,14]$.

Tensor force is one of the most important ingredients of $N N$ interactions in nuclei, as manifested by the quadruple moment of deuteron [15]. Over the past decades, the effects of tensor force in exotic neutron-rich nuclei have also attracted a lot of attention due to its essential role in the evolution of shell structure [16-20]. Moreover, the tensor-force effects in nuclear excitations have also been investigated extensively, such as Gamow-Teller transitions [21], charge-exchange spindipole excitations [22], non-charge-exchange multipole responses [23], the first $0^{-}$excitation energies [24], and magnetic excitations [25]. Lots of efforts have been devoted to explore the impact of tensor forces in nonrelativistic Skyrme

*pwzhao@pku.edu.cn
[26-38] and Gogny [18,24,39-42] density functional theories (DFTs) as well as the relativistic ones [19,43-51].

However, the determination of the strength of tensor forces in finite nuclei is very challenging because it is difficult to find significant features in experimental data which are only connected to tensor forces and therefore suitable for an adjustment of their parameters [13]. Therefore, much attention has been paid to the metadata from microscopic ab initio calculations for constraining the strength of tensor forces.

The neutron drop consisting of a certain number of neutrons confined in an external potential can be calculated with both $a b$ initio methods [52-59] and DFTs [60,61], due to the simplicity of missing proton-neutron interactions. In Ref. [61], strong linear correlations between the neutron drop radii and the neutron skin thickness have been established, and the linear correlations are used to constrain the three-neutron forces.

In Ref. [13], the tensor-force effect in neutron drops has been studied by the $a b$ initio RBHF theory with the Bonn-A interaction [62]. Moreover, it is suggested that the strength of tensor force in the relativistic Hartree-Fock (RHF) density functional PKO1 [43] could be determined by adjusting to the RBHF results for the evolution of SO splittings in the neutron drops from $N=8$ to $N=50$ confined in a harmonic trap with the strength $\hbar \omega=10 \mathrm{MeV}$. This provides an interesting way to determine the strength of tensor forces in nuclear density functionals, and it has been implemented in recent works $[13,14,63]$.

In Refs. $[13,14,63]$, the strength $\hbar \omega$ for the external harmonic oscillator (HO) field in neutron drops is fixed at $\hbar \omega=$ $10 \mathrm{MeV}$. In Refs. [61] and [64], the nuclear matter properties at saturation density and two times saturation density have been investigated, by choosing the strength $\hbar \omega=10$ and 
$25 \mathrm{MeV}$, respectively. Therefore, it is important to investigate the impact of the strength of external fields for neutron drops on the tensor-force strength.

In this work, the neutron drops with even neutron number from $N=8$ to $N=50$ confined in a harmonic trap with the strength varying from $\hbar \omega=5$ to $\hbar \omega=20 \mathrm{MeV}$ are studied with the RBHF theory and RHF theory. In particular, the impact of tensor forces is investigated by analyzing the evolution of SO splittings of neutron drops in different external fields.

This paper is organized as follows. In Sec. II, the theoretical framework of the RBHF and RHF theories is briefly introduced. The numerical details are given in Sec. III. The calculated results and the discussions are presented in Sec. IV. Finally, the summary is given in Sec. V.

\section{THEORETICAL FRAMEWORK}

Starting from a relativistic one-boson-exchange $N N$ interaction [62], one can build the stationary many-body Hamiltonian,

$$
\begin{aligned}
H= & \int d^{3} \boldsymbol{r} \bar{\psi}(-i \boldsymbol{\gamma} \cdot \nabla+M) \psi \\
& +\frac{1}{2} \sum_{\alpha} \int d^{3} \boldsymbol{r}_{1} d^{3} \boldsymbol{r}_{2} \bar{\psi}\left(\boldsymbol{r}_{1}\right) \Gamma_{\alpha}^{(1)} \psi\left(\boldsymbol{r}_{1}\right) D_{\alpha}\left(\boldsymbol{r}_{1}, \boldsymbol{r}_{2}\right) \bar{\psi}\left(\boldsymbol{r}_{2}\right) \\
& \times \Gamma_{\alpha}^{(2)} \psi\left(\boldsymbol{r}_{2}\right)
\end{aligned}
$$

where $\psi$ denotes the nucleon field and $M$ is the rest mass of the nucleon. The index $\alpha$ is running over all types of mesons to be exchanged in the $N N$ interaction and the exchanged photons in the Coulomb interaction. The interaction vertices $\Gamma_{\alpha}^{(1)}$ and $\Gamma_{\alpha}^{(2)}$ correspond to the particles 1 and 2 with the coordinates $\boldsymbol{r}_{1}$ and $\boldsymbol{r}_{2}$, respectively. The retardation effects are ignored in the propagator $D_{\alpha}\left(\boldsymbol{r}_{1}, \boldsymbol{r}_{2}\right)$ for mesons and photons. For the Bonn interactions, each vertex is attached with a form factor of monopole type [62].

The nucleon field $\psi(\boldsymbol{r})$ can be expanded with a given set of Dirac spinors $\psi_{k}(\boldsymbol{r})$ :

$$
\psi^{\dagger}(\boldsymbol{r})=\sum_{k} \psi_{k}^{\dagger}(\boldsymbol{r}) b_{k}^{\dagger}, \quad \psi(\boldsymbol{r})=\sum_{k} \psi_{k}(\boldsymbol{r}) b_{k},
$$

where $b_{k}^{\dagger}$ and $b_{k}$ form a complete set of creation and annihilation operators for nucleons in the state $|k\rangle$ with positive or negative energies. Then, the Hamiltonian (1) can be rewritten as

$$
H=\sum_{k l}\langle k|T| l\rangle b_{k}^{\dagger} b_{l}+\frac{1}{2} \sum_{\alpha} \sum_{k l m n}\left\langle k l\left|V_{\alpha}\right| m n\right\rangle b_{k}^{\dagger} b_{l}^{\dagger} b_{n} b_{m},
$$

where the relativistic kinetic energy matrix element $\langle k|T| l\rangle$ and bare $N N$ interaction matrix elements $\left\langle k l\left|V_{\alpha}\right| m n\right\rangle$ are, respectively, given by

$$
\begin{aligned}
\langle k|T| l\rangle= & \int d^{3} \boldsymbol{r} \bar{\psi}_{k}(\boldsymbol{r})(-i \boldsymbol{\gamma} \cdot \nabla+M) \psi_{l}(\boldsymbol{r}) \\
\left\langle k l\left|V_{\alpha}\right| m n\right\rangle= & \int d^{3} \boldsymbol{r}_{1} d^{3} \boldsymbol{r}_{2} \bar{\psi}_{k}\left(\boldsymbol{r}_{1}\right) \Gamma_{\alpha}^{(1)} \psi_{m}\left(\boldsymbol{r}_{1}\right) D_{\alpha}\left(\boldsymbol{r}_{1}, \boldsymbol{r}_{2}\right) \bar{\psi}_{l}\left(\boldsymbol{r}_{2}\right) \\
& \times \Gamma_{\alpha}^{(2)} \psi_{n}\left(\boldsymbol{r}_{2}\right) .
\end{aligned}
$$

Due to the strong repulsive core in the bare $N N$ interaction, a direct Hartree-Fock solution of the Hamiltonian (3) is not accessible. The Brueckner theory $[65,66]$ is, thus, adopted to soften the bare interaction into an effective interaction in the nuclear medium, i.e., the $G$ matrix. It takes into account the short-range correlations by summing up all the ladder diagrams of the bare interaction and is deduced from the Bethe-Goldstone equation [67]

$$
\begin{aligned}
\left\langle a b|\bar{G}(W)| a^{\prime} b^{\prime}\right\rangle= & \left\langle a b|\bar{V}| a^{\prime} b^{\prime}\right\rangle+\frac{1}{2} \sum_{c d}\langle a b|\bar{V}| c d\rangle \\
& \times \frac{Q(c, d)}{W-\varepsilon_{c}-\varepsilon_{d}}\left\langle c d|\bar{G}(W)| a^{\prime} b^{\prime}\right\rangle,
\end{aligned}
$$

where $\left\langle a b|\bar{V}| a^{\prime} b^{\prime}\right\rangle=\sum_{\alpha}\left\langle a b\left|V_{\alpha}\right| a^{\prime} b^{\prime}-b^{\prime} a^{\prime}\right\rangle$ is the antisymmetrized two-body matrix element, and the summation of $\alpha$ is only running over mesons. The starting energy is denoted by $W$, and $\varepsilon_{c}$ and $\varepsilon_{d}$ represent the single-particle energies of the $|c\rangle$ and $|d\rangle$, respectively. The Pauli operator $Q(c, d)$ allows the intermediate states $c$ and $d$ in Eq. (6) to run over all the states above the Fermi surface.

In the relativistic framework, the single-particle states are obtained by solving the RHF equation

$$
\left(T+U+\frac{1}{2} M \omega^{2} r^{2}\right)|a\rangle=e_{a}|a\rangle,
$$

where $e_{a}=\varepsilon_{a}+M$ is the single-particle energy with the rest mass of nucleon $M$. Here, the external field $U_{\text {ex }}=$ $\frac{1}{2} M \omega^{2} r^{2}$ has been added explicitly for neutron drops. The self-consistent single-particle potential $U$ is defined by the $G$ matrix

$$
\langle a|U| b\rangle=\sum_{c=1}^{A}\langle a c|\bar{G}(W)| b c\rangle,
$$

where the index $c$ runs over all the occupied states in the Fermi sea (no-sea approximation). The detailed choices of the starting energy $W$ can be found in Refs. [12,14].

In contrast to the RBHF theory, the starting point of the RHF theory is an effective interaction, which is intermediated by two isoscalar meson fields ( $\sigma$ and $\omega$ ), two isovector meson fields ( $\pi$ and $\rho$ ), and the photon field $A$, and is adjusted to the properties of finite nuclei and nuclear matter [43]. The selfconsistent single-particle potential comes directly from this effective interaction, rather than from $G$ matrix in the RBHF theory. Details can be found in Refs. [43,68].

\section{NUMERICAL DETAILS}

For the RBHF calculations, the Bonn-A [62] interaction is used in this work. The initial single-particle basis is chosen as a set of Dirac Woods-Saxon basis [69], and it is updated self-consistently in each iteration of the RBHF calculations [12]. The energy cut-off is $900 \mathrm{MeV}$ for the positive-energy states and $-1700 \mathrm{MeV}$ for the negative-energy ones, and the single-particle orbital angular momentum cut-off is $25 \hbar$. The two-particle coupled total angular momentum cut-off is $10 \hbar$. Spherical symmetry is assumed in the calculations, and the external HO fields with $\hbar \omega=5,10,15$, and $20 \mathrm{MeV}$ are 

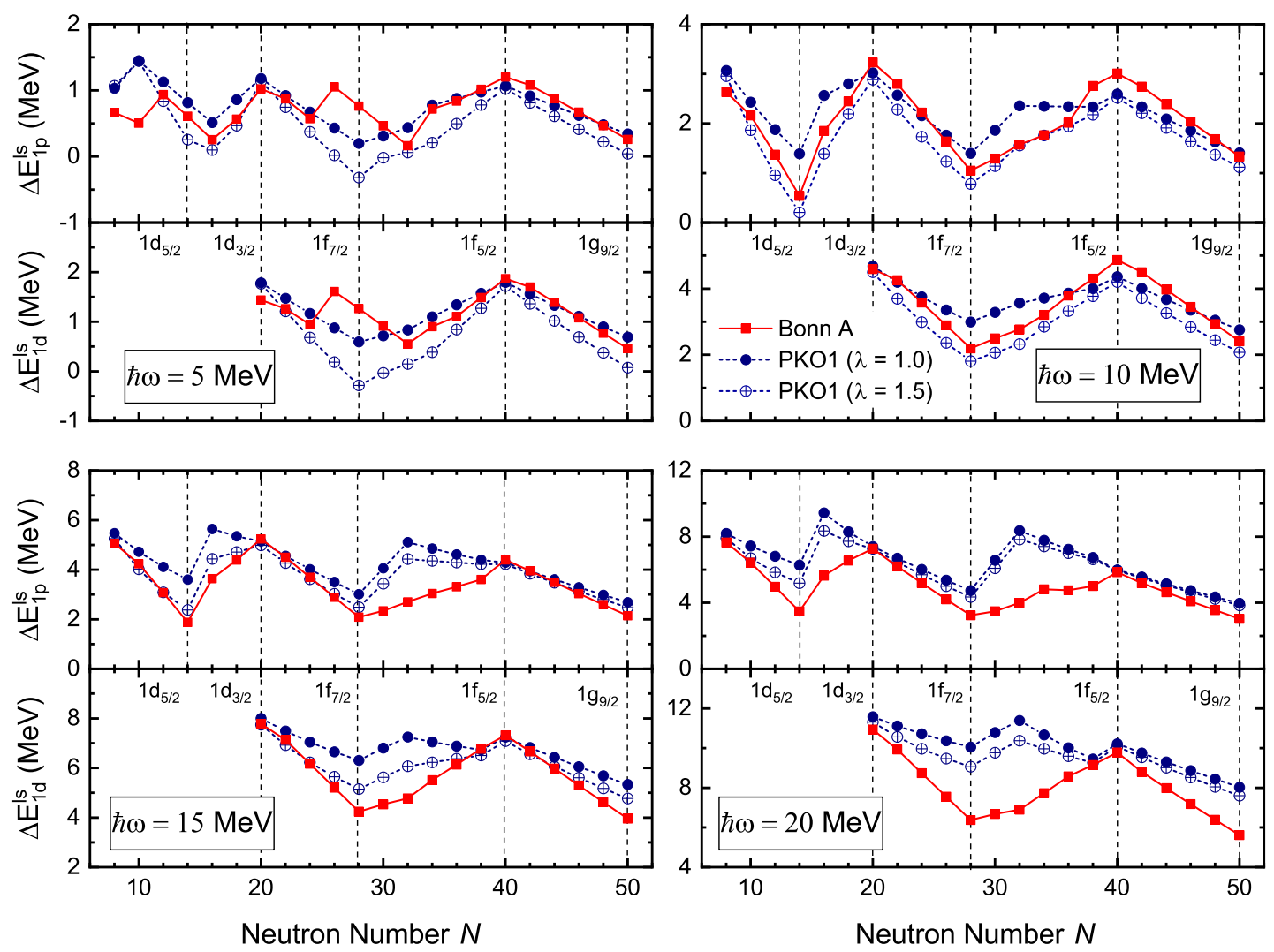

FIG. 1. The evolution of the $1 p$ and $1 d$ spin-orbit splittings with the neutron number $N$ for neutron drops in a $\mathrm{HO}$ trap with $\hbar \omega=5$, 10 , $15,20 \mathrm{MeV}$ calculated by RBHF theory using the Bonn-A [62] interaction, in comparison with the results obtained by RHF density functional PKO1 [43] with different strength of $\pi N$ coupling characterized by $\lambda$.

considered for neutron drops. The Dirac basis is solved in a box with the size $R_{\mathrm{box}}=7 \mathrm{fm}$ for $\hbar \omega=15$ and $20 \mathrm{MeV}$, and $R_{\text {box }}=8 \mathrm{fm}$ for $\hbar \omega=5$ and $10 \mathrm{MeV}$.

For the RHF calculations, the effective interaction PKO1 is adopted [43]. The $\pi N$ coupling constant $f_{\pi}$ has an explicit exponential density-dependent form

$$
f_{\pi}\left(\rho_{b}\right)=f_{\pi}(0) e^{-a_{\pi} \xi}
$$

where $\xi=\rho_{b} / \rho_{0}$ is the ratio of baryonic density $\rho_{b}$ over baryonic saturation density of nuclear matter $\rho_{0}=$ $0.1520 \mathrm{fm}^{-3}$ and $a_{\pi}=1.2320, f_{\pi}(0)=1.0$. In the following, as in Ref. [13], a factor $\lambda$ is introduced in front of $f_{\pi}(0)$ as a strength parameter.

\section{RESULTS AND DISCUSSION}

In Fig. 1 , the evolution of the $1 p$ and $1 d$ SO splittings with the neutron number $N$ for neutron drops in a HO trap with $\hbar \omega=5,10,15,20 \mathrm{MeV}$ is depicted. As pointed out in Ref. [13], the zigzag behavior of the SO splittings obtained with the RBHF theory can be attributed to the tensor-force mechanism proposed by Otsuka et al. [17], i.e., nucleons in two SO aligned orbits or two SO antialigned orbits are repulsive, while nucleons in two orbits with opposite $\mathrm{SO}$ alignment are attractive. For instance, neutrons are filling in the SO aligned orbit $1 f_{7 / 2}$ from $N=20$ to $N=28$. This occupation could shift upward the $1 d_{5 / 2}$ orbit and downward the $1 d_{3 / 2}$ one due to the tensor-force mechanism and, thus, reduce the $1 d$ SO splittings. Above $N=28$, the $\mathrm{SO}$ antialigned orbits $2 p_{1 / 2}$ and $1 f_{5 / 2}$ are filled. This occupation shifts downward the $1 d_{5 / 2}$ orbit and upward the $1 d_{3 / 2}$ one and, thus, leads to the increase of the $1 d$ SO splittings. Therefore, the RBHF results for the SO splittings in neutron drops provide very useful constraints for determining the tensor-force strength in the phenomenological RHF calculations.

As compared to the RBHF SO splittings for the neutron drops in a $\mathrm{HO}$ field with $\hbar \omega=10 \mathrm{MeV}$ (see the upper right panel of Fig. 1), the tensor-force effect is somewhat too weak in the corresponding RHF calculations $(\lambda=1.0)$ though similar behavior could be obtained. Therefore, it was suggested in Ref. [13] that the strength parameter $\lambda$ should be enlarged. Here, for example, by adjusting $\lambda$ to 1.5 , the zigzag behavior of the SO splittings obtained with RBHF can be well reproduced. Note that although we present here the results for $\lambda=1.5$ rather than 1.3 as in Ref. [13], it does not mean that $\lambda=1.5$ must be a better choice than $\lambda=1.3$. In fact, what has been done currently is solely adjusting $\lambda$ to show the feasibility of determining tensor-force strength from microscopic calculations and careful fitting work is needed to get the precise values.

On the other hand, to perform such a fitting work, it is very necessary to study how the value of $\lambda$ is influenced by the external fields of neutron drops. In the upper left panel of Fig. 1, the RBHF and RHF SO splittings are compared for neutron 


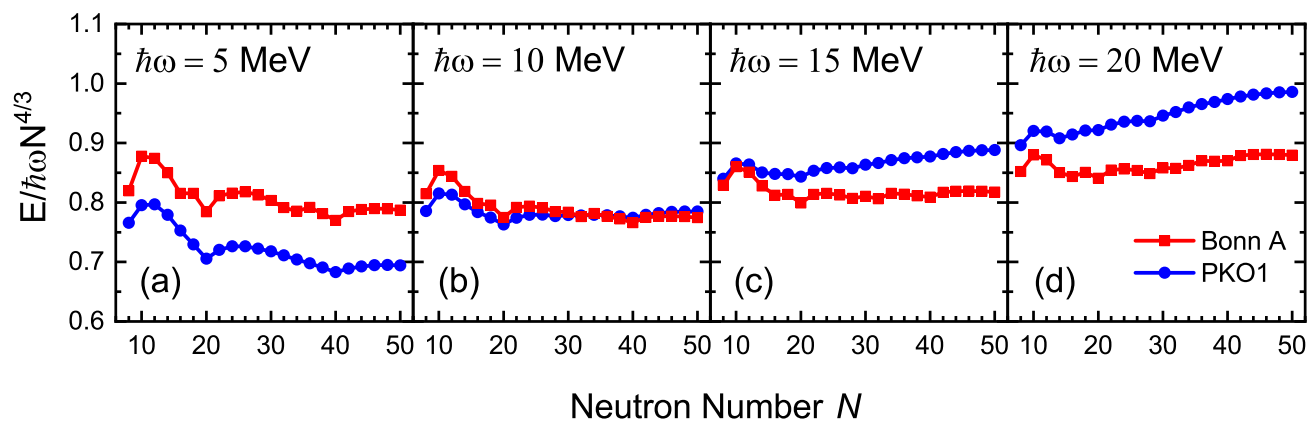

FIG. 2. Renormalized total energies by $\hbar \omega N^{4 / 3}$ for $N$-neutron drops in a HO trap $(\hbar \omega=5,10,15,20 \mathrm{MeV})$ calculated by RBHF theory using the interaction Bonn A in comparison with the results obtained by the RHF approach with PKO1.

drops in a $\mathrm{HO}$ field with $\hbar \omega=5 \mathrm{MeV}$. Generally speaking, the RHF results with $\lambda=1.0$ are in a better agreement with the corresponding RBHF ones. Note that here the bumps of $1 p$ and $1 d$ SO splittings at $N=26$ in RBHF calculations are not caused by tensor forces but by the energy level inversion of $2 p_{3 / 2}$ and $1 f_{7 / 2}$.

The results for neutron drops in HO fields with $\hbar \omega=$ $15 \mathrm{MeV}$ and $\hbar \omega=20 \mathrm{MeV}$ are presented in the lower left and lower right panels of Fig. 1, respectively. In the case of $\hbar \omega=15 \mathrm{MeV}$, the RHF SO splittings are in general larger than the RBHF ones, and the corresponding zigzag tendency is weaker as well. This indicates that the strength parameter $\lambda$ should be in general slightly larger than 1.5 to reproduce the RBHF results for neutron drops with $\hbar \omega=15 \mathrm{MeV}$. Several exceptions occur, however, in the drops with neutron number $8 \leqslant N \leqslant 14$, where the RHF results with $\lambda=1.5$ can well reproduce the RBHF ones. Furthermore, in the case of $\hbar \omega=20 \mathrm{MeV}$, it is clear that the RHF results with even $\lambda=1.5$ deviate significantly from the RBHF ones, and this indicates that a much larger strength parameter $\lambda$ is needed. It becomes clear now that the value of the optimized $\lambda$ in RHF is running with the strength $\hbar \omega$ of the external fields of neutron drops.

Furthermore, other neutron drop properties are also investigated. In Fig. 2, the renormalized total energies by $\hbar \omega N^{4 / 3}$ obtained with RBHF and RHF theories for neutron drops with the neutron number $N$ from 8 to 50 in a $\mathrm{HO}$ trap with $\hbar \omega=5$, $10,15,20 \mathrm{MeV}$ are depicted. For a given neutron drop, the renormalized total energies obtained with the RBHF theory do not depend on the variation of external fields considerably, but the ones obtained with RHF exhibit a strong $\hbar \omega$ dependence. Specifically, the RHF energies become higher and higher with the increasing strength of the external fields from $\hbar \omega=5$ to $20 \mathrm{MeV}$. A good consistency between the RHF and RBHF results can only be found in the case of $\hbar \omega=10 \mathrm{MeV}$ for the neutron number $N>14$ as well as the case of $\hbar \omega=15 \mathrm{MeV}$ for $8 \leqslant N \leqslant 14$. It should be noted that the strength of the external fields in neutron drops essentially influences the density of the system. Therefore, the varying discrepancies between the RHF and RBHF results in different external fields indicate that the phenomenological density-dependent behavior of the effective interaction PKO1 is not compatible with the $G$ matrix derived from the realistic interaction Bonn $\mathrm{A}$ in the ab initio RBHF theory.
By taking the neutron drops with neutron number $N=$ 8, 20, 40 as examples, in Fig. 3, the renormalized density distributions by saturation density $\rho_{0}$ of nuclear matter are depicted. Note that the saturation density $\rho_{0}$ obtained in the RBHF theory with Bonn A is $0.180 \mathrm{fm}^{-3}$ [70], while it is $0.152 \mathrm{fm}^{-3}$ [43] in the RHF calculations with PKO1.

For $\hbar \omega=5 \mathrm{MeV}$, in both RHF and RBHF calculations, the central densities of all neutron drops considered here are much lower than $\rho_{0}$. Since the RHF density functional PKO1 is mainly adjusted to the ground-state properties of several finite nuclei, it is loosely constrained in the low- and high-density regions. In the RBHF calculations, however, the $G$ matrix represents the $N N$ interaction in nuclear medium and it is derived microscopically from the realistic interaction Bonn A determined by fitting to $N N$ scattering data with a high precision. Therefore, it is not surprising at all that the RHF and RBHF total energies for $\hbar \omega=5 \mathrm{MeV}$ differ dramatically, as shown in Fig. 2(a).

For $\hbar \omega=10 \mathrm{MeV}$, the central densities of neutron drops with $N=20$ and 40 in both the RHF and RBHF calculations are very close to $\rho_{0}$, i.e., around $0.9 \rho_{0}$. Since the RHF density functional PKO1 is nicely determined around $\rho_{0}$ by fitting to nuclear ground-state properties, it is expected that the RHF total energies for neutron drops with the central densities close to $\rho_{0}$ are consistent with the corresponding RBHF results;

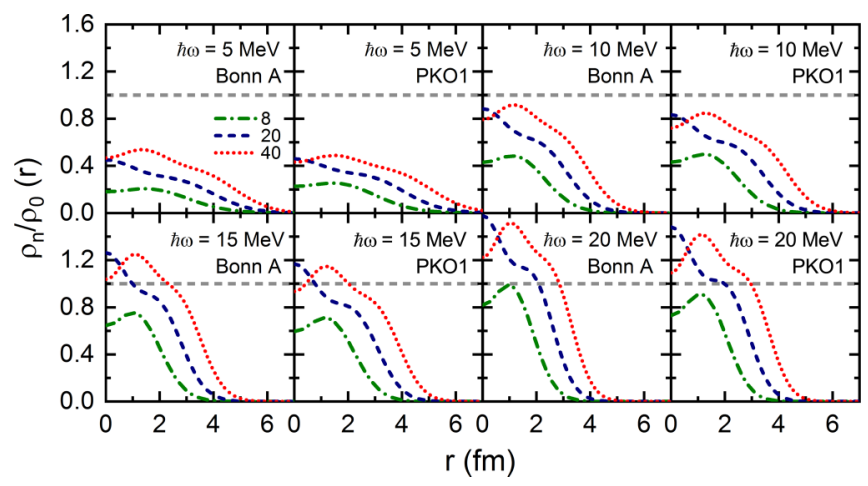

FIG. 3. Renormalized density distributions by nuclear matter saturation density $\rho_{0}$ for $N$-neutron drops in a $\mathrm{HO} \operatorname{trap}(\hbar \omega=$ $5,10,15,20 \mathrm{MeV}$ ) calculated by RBHF theory using the interaction Bonn A in comparison with the results obtained by the RHF approach with PKO1. The gray dashed lines denote the position of $\rho_{0}$. 


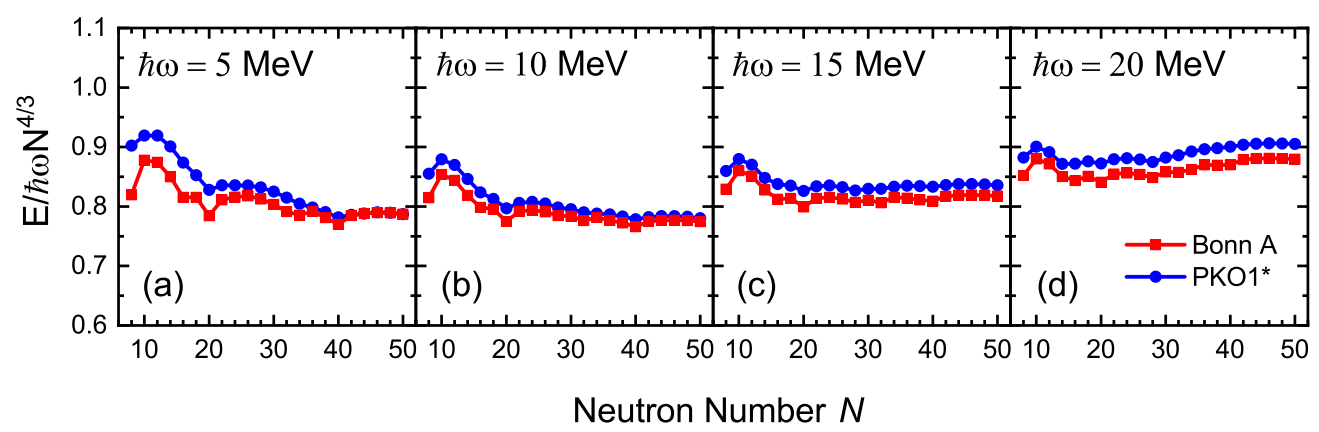

FIG. 4. Same as Fig. 2, but for a rescaled density dependence of the RHF density functional.

see Fig. 2(b). Note that the central densities of the neutron drop with $N=8$ are around $0.5 \rho_{0}$ in both RHF and RBHF calculations for $\hbar \omega=10 \mathrm{MeV}$. This value is still a little bit far away from $\rho_{0}$, and this may explain the discrepancies between the RHF and RBHF total energies for $8 \leqslant N \leqslant 14$ shown in Fig. 2(b).

For $\hbar \omega=15 \mathrm{MeV}$, the central densities of the neutron drop with $N=8$ are getting larger as compared to the case with $\hbar \omega=10 \mathrm{MeV}$. Accordingly, a better agreement between the RHF and RBHF total energies for $8 \leqslant N \leqslant 14$ is obtained, as shown in Fig. 2(c). For the neutron drops with $N=20$ and 40, however, the central densities are evidently higher than $\rho_{0}$ in both RHF and RBHF calculations. Again, due to the fact that the RHF density functional PKO1 is not well constrained in the supradensity region, the discrepancies between the RHF and RBHF total energies for $N \geqslant 16$ shown in Fig. 2(c) are justified.

For $\hbar \omega=20 \mathrm{MeV}$, the central densities of the neutron drops with $N=20$ and 40 are significantly larger than $\rho_{0}$, and this is connected with the substantial differences between the RHF and RBHF total energies observed in Fig. 2(d). While the central densities of the neutron drop with $N=8$ are around $\rho_{0}$, the agreement between the RHF and RBHF total energies is less pronounced as shown in Fig. 2(d). This might be related to the fact that the interior part of light nuclear systems usually cannot be regarded as a fully saturated nuclear matter.

Therefore, it is clear that to perform a microscopic derivation of nuclear energy density functionals with tensor forces via neutron drops without rescaling the density dependence of functionals, it is crucial to assure the central densities of neutron drops close to $\rho_{0}$.

In addition, to investigate if the $\hbar \omega$ dependence of $\lambda$ is due to the inconsistent density dependence between the effective interaction PKO1 and the $a b$ initio $G$ matrix, the density dependence of the RHF density functional is rescaled to the equation of states of symmetric nuclear matter and pure neutron matter calculated by the RBHF theory with Bonn A [71]. In Fig. 4, the renormalized total energies for neutron drops obtained with the rescaled density-dependent RHF density functional (labeled as $\mathrm{PKO} 1^{*}$ ) are depicted. It is found that a good consistency between the RHF and RBHF total energies can be achieved in different external fields. Nevertheless, to reproduce the RBHF SO splittings, one still needs to adjust the tensor-force strength factor $\lambda$, and the optimized $\lambda=8$, 10,15 , and 20 are obtained for $\hbar \omega=5,10,15$, and $20 \mathrm{MeV}$, respectively. Therefore, the optimized $\lambda$ is still running with the external fields, and this reflects the density-dependent nature of the tensor-force strength $\lambda$.

Therefore, it could be also interesting, in the future, to determine the density dependence of effective interactions in nuclear energy density functionals by adjusting to the inhomogeneous neutron drop properties in various external fields extracted from $a b$ initio calculations.

\section{SUMMARY}

In summary, the neutron drops with even neutron number $N$ from 8 to 50 confined in a harmonic oscillator trap with $\hbar \omega=5,10,15$, and $20 \mathrm{MeV}$ are studied with the relativistic Brueckner-Hartree-Fock and relativistic Hartree-Fock theories. The evolution of spin-orbit splittings along with the neutron number has been investigated and its connection with the tensor-force strength has been analyzed systematically for different external fields of the neutron drops. For the RHF density functional PKO1, the optimized tensor-force strength $\lambda$ in the RHF theory, which reproduces the microscopic RBHF spin-orbit splittings, is running with the strength of the external fields of neutron drops, and the total energies calculated with the RBHF and RHF theories are consistent only for certain neutron drops. To guarantee that the optimized $\lambda$ corresponds to the tensor-force effects for the evolution of spin-orbit splittings in neutron drops, one has to determine a reasonable external field to obtain the consistent RBHF and RHF total energies. This could be useful to guide the future microscopic derivations of nuclear energy density functionals with tensor forces. A good choice could be to assure the central densities of neutron drops close to nuclear matter saturation density, for instance, $\hbar \omega=10 \mathrm{MeV}$ for neutron number $N>14$ and $\hbar \omega=15 \mathrm{MeV}$ for $8 \leqslant N \leqslant 14$. On the other hand, if one rescales the density dependence of the RHF functional, a good consistency between the RBHF and RHF results for the total energies of various neutron drops can be achieved. Even so, the optimized tensor-force strength $\lambda$ is still running with the strength of the external fields of neutron drops. This reflects the density-dependent nature of the tensor-force strength $\lambda$.

\section{ACKNOWLEDGMENTS}

We thank Qiang Zhao for discussions. This work was supported in part by the National Key R\&D Pro- 
gram of China (Contracts No. 2018YFA0404400 and No. 2017YFE0116700), the National Natural Science Foundation of China (Grants No. 11775026, No. 11875075, No.
11621131001, No. 11935003, and No. 11975031), and the Laboratory Computing Resource Center at Argonne National Laboratory.
[1] P. Ring, Prog. Part. Nucl. Phys. 37, 193 (1996).

[2] D. Vretenar, A. V. Afanasjev, G. A. Lalazissis, and P. Ring, Phys. Rep. 409, 101 (2005).

[3] J. Meng, H. Toki, S.-G. Zhou, S. Q. Zhang, W. H. Long, and L. S. Geng, Prog. Part. Nucl. Phys. 57, 470 (2006).

[4] T. Nikšić, D. Vretenar, and P. Ring, Prog. Part. Nucl. Phys. 66, 519 (2011)

[5] J. Meng and S.-G. Zhou, J. Phys. G 42, 093101 (2015).

[6] H. Z. Liang, J. Meng, and S.-G. Zhou, Phys. Rep. 570, 1 (2015).

[7] Relativistic Density Functional for Nuclear Structure, edited by J. Meng (World Scientific, Singapore, 2015).

[8] J. E. Drut, R. J. Furnstahl, and L. Platter, Prog. Part. Nucl. Phys. 64, 120 (2010).

[9] S. H. Shen, H. Z. Liang, W. H. Long, J. Meng, and P. Ring, Prog. Part. Nucl. Phys. 109, 103713 (2019).

[10] X. Roca-Maza, X. Viñas, M. Centelles, P. Ring, and P. Schuck, Phys. Rev. C 84, 054309 (2011).

[11] S. Shen, J. Hu, H. Liang, J. Meng, P. Ring, and S. Zhang, Chin. Phys. Lett. 33, 102103 (2016).

[12] S. Shen, H. Liang, J. Meng, P. Ring, and S. Zhang, Phys. Rev. C 96, 014316 (2017).

[13] S. Shen, H. Liang, J. Meng, P. Ring, and S. Zhang, Phys. Lett. B 778, 344 (2018).

[14] S. Shen, H. Liang, J. Meng, P. Ring, and S. Zhang, Phys. Rev. C 97, 054312 (2018).

[15] H. A. Bethe, Phys. Rev. 57, 390 (1940).

[16] T. Otsuka, R. Fujimoto, Y. Utsuno, B. A. Brown, M. Honma, and T. Mizusaki, Phys. Rev. Lett. 87, 082502 (2001).

[17] T. Otsuka, T. Suzuki, R. Fujimoto, H. Grawe, and Y. Akaishi, Phys. Rev. Lett. 95, 232502 (2005).

[18] T. Otsuka, T. Matsuo, and D. Abe, Phys. Rev. Lett. 97, 162501 (2006).

[19] W. H. Long, H. Sagawa, N. V. Giai, and J. Meng, Phys. Rev. C 76, 034314 (2007).

[20] T. Otsuka, T. Suzuki, M. Honma, Y. Utsuno, N. Tsunoda, K. Tsukiyama, and M. Hjorth-Jensen, Phys. Rev. Lett. 104, 012501 (2010).

[21] C. L. Bai, H. Sagawa, H. Q. Zhang, X. Z. Zhang, G. Colò, and F. R. Xu, Phys. Lett. B 675, 28 (2009).

[22] C. L. Bai, H. Q. Zhang, H. Sagawa, X. Z. Zhang, G. Colò, and F. R. Xu, Phys. Rev. Lett. 105, 072501 (2010).

[23] L. G. Cao, G. Colò, H. Sagawa, P. F. Bortignon, and L. Sciacchitano, Phys. Rev. C 80, 064304 (2009).

[24] M. Anguiano, G. Co', V. De Donno, and A. M. Lallena, Phys. Rev. C 83, 064306 (2011).

[25] G. Co', V. De Donno, M. Anguiano, and A. M. Lallena, Phys. Rev. C 85, 034323 (2012).

[26] F. Stancu, D. M. Brink, and H. Flocard, Phys. Lett. B 68, 108 (1977).

[27] B. A. Brown, T. Duguet, T. Otsuka, D. Abe, and T. Suzuki, Phys. Rev. C 74, 061303(R) (2006).

[28] G. Colò, H. Sagawa, S. Fracasso, and P. F. Bortignon, Phys. Lett. B 646, 227 (2007).

[29] D. M. Brink and F. Stancu, Phys. Rev. C 75, 064311 (2007).
[30] T. Lesinski, M. Bender, K. Bennaceur, T. Duguet, and J. Meyer, Phys. Rev. C 76, 014312 (2007).

[31] W. Zou, G. Colò, Z. Y. Ma, H. Sagawa, and P. F. Bortignon, Phys. Rev. C 77, 014314 (2008).

[32] M. Zalewski, J. Dobaczewski, W. Satuła, and T. R. Werner, Phys. Rev. C 77, 024316 (2008).

[33] M. Moreno-Torres, M. Grasso, H. Z. Liang, V. De Donno, M. Anguiano, and N. Van Giai, Phys. Rev. C 81, 064327 (2010).

[34] V. Hellemans, P.-H. Heenen, and M. Bender, Phys. Rev. C 85, 014326 (2012).

[35] X. Roca-Maza, G. Colò, and H. Sagawa, Phys. Scr. T154, 014011 (2013).

[36] M. Grasso, Phys. Rev. C 89, 034316 (2014).

[37] E. Yüksel, N. Van Giai, E. Khan, and K. Bozkurt, Phys. Rev. C 89, 064322 (2014).

[38] H. Sagawa and G. Colò, Prog. Part. Nucl. Phys. 76, 76 (2014).

[39] M. Anguiano, M. Grasso, G. Co', V. De Donno, and A. M. Lallena, Phys. Rev. C 86, 054302 (2012).

[40] M. Grasso and M. Anguiano, Phys. Rev. C 88, 054328 (2013).

[41] M. Anguiano, A. M. Lallena, G. Co', V. De Donno, M. Grasso, and R. N. Bernard, Eur. Phys. J. A 52, 183 (2016).

[42] H. Nakada, K. Sugiura, and J. Margueron, Phys. Rev. C 87, 067305 (2013).

[43] W.-H. Long, N. Van Giai, and J. Meng, Phys. Lett. B 640, 150 (2006).

[44] W. H. Long, H. Sagawa, J. Meng, and N. Van Giai, Europhys. Lett. 82, 12001 (2008).

[45] D. Tarpanov, H. Liang, N. V. Giai, and C. Stoyanov, Phys. Rev. C 77, 054316 (2008).

[46] G. A. Lalazissis, S. Karatzikos, M. Serra, T. Otsuka, and P. Ring, Phys. Rev. C 80, 041301(R) (2009).

[47] S. Marcos, M. López-Quelle, R. Niembro, and L. N. Savushkin, Phys. At. Nucl. 77, 299 (2014).

[48] L. J. Jiang, S. Yang, B. Y. Sun, W. H. Long, and H. Q. Gu, Phys. Rev. C 91, 034326 (2015).

[49] J. J. Li, J. Margueron, W. H. Long, and N. Van Giai, Phys. Lett. B 753, 97 (2016).

[50] K. Karakatsanis, G. A. Lalazissis, P. Ring, and E. Litvinova, Phys. Rev. C 95, 034318 (2017).

[51] Z. Wang, Q. Zhao, H. Liang, and W. H. Long, Phys. Rev. C 98, 034313 (2018).

[52] B. S. Pudliner, A. Smerzi, J. Carlson, V. R. Pandharipande, S. C. Pieper, and D. G. Ravenhall, Phys. Rev. Lett. 76, 2416 (1996).

[53] A. Smerzi, D. G. Ravenhall, and V. R. Pandharipande, Phys. Rev. C 56, 2549 (1997).

[54] F. Pederiva, A. Sarsa, K. Schmidt, and S. Fantoni, Nucl. Phys. A 742, 255 (2004).

[55] S. Gandolfi, J. Carlson, and S. C. Pieper, Phys. Rev. Lett. 106, 012501 (2011).

[56] S. K. Bogner, R. J. Furnstahl, H. Hergert, M. Kortelainen, P. Maris, M. Stoitsov, and J. P. Vary, Phys. Rev. C 84, 044306 (2011).

[57] P. Maris, J. P. Vary, S. Gandolfi, J. Carlson, and S. C. Pieper, Phys. Rev. C 87, 054318 (2013). 
[58] H. D. Potter, S. Fischer, P. Maris, J. P. Vary, S. Binder, A. Calci, J. Langhammer, and R. Roth, Phys. Lett. B 739, 445 (2014).

[59] I. Tews, S. Gandolfi, A. Gezerlis, and A. Schwenk, Phys. Rev. C 93, 024305 (2016).

[60] M. Kortelainen, J. McDonnell, W. Nazarewicz, E. Olsen, P.-G. Reinhard, J. Sarich, N. Schunck, S. M. Wild, D. Davesne, J. Erler, and A. Pastore, Phys. Rev. C 89, 054314 (2014).

[61] P. W. Zhao and S. Gandolfi, Phys. Rev. C 94, 041302(R) (2016).

[62] R. Machleidt, in Advances in Nuclear Physics, Vol. 19, edited by J. W. Negele and E. Vogt (Springer, New York, 1989), pp. 189-376.

[63] S. Shen, G. Colò, and X. Roca-Maza, Phys. Rev. C 99, 034322 (2019).
[64] H. Tong, P.-W. Zhao, and J. Meng, arXiv:1903.05938v1 [nucl-th].

[65] K. A. Brueckner, C. A. Levinson, and H. M. Mahmoud, Phys. Rev. 95, 217 (1954).

[66] K. A. Brueckner, Phys. Rev. 96, 508 (1954).

[67] H. A. Bethe and J. Goldstone, Proc. R. Soc. London, Ser. A 238 , 551 (1957).

[68] A. Bouyssy, J.-F. Mathiot, N. Van Giai, and S. Marcos, Phys. Rev. C 36, 380 (1987)

[69] S.-G. Zhou, J. Meng, and P. Ring, Phys. Rev. C 68, 034323 (2003).

[70] H. Tong, X.-L. Ren, P. Ring, S.-H. Shen, S.-B. Wang, and J. Meng, Phys. Rev. C 98, 054302 (2018).

[71] E. N. E. van Dalen, C. Fuchs, and A. Faessler, Eur. Phys. J. A 31, 29 (2007). 